\title{
A Weak Solution of a Stochastic Nonlinear Problem
}

\author{
M. L. Hadji \\ Laboratory of Probability and Statistics (LAPS), Badji Mokhtar University, P.O. Box 12, 23000 Annaba, Algeria
}

Correspondence should be addressed to M. L. Hadji; mohamedlakhdarhadji@gmail.com

Received 3 October 2014; Accepted 25 December 2014

Academic Editor: Jaan Janno

Copyright (C) 2015 M. L. Hadji. This is an open access article distributed under the Creative Commons Attribution License, which permits unrestricted use, distribution, and reproduction in any medium, provided the original work is properly cited.

We consider a problem modeling a porous medium with a random perturbation. This model occurs in many applications such as biology, medical sciences, oil exploitation, and chemical engineering. Many authors focused their study mostly on the deterministic case. The more classical one was due to Biot in the 50s, where he suggested to ignore everything that happens at the microscopic level, to apply the principles of the continuum mechanics at the macroscopic level. Here we consider a stochastic problem, that is, a problem with a random perturbation. First we prove a result on the existence and uniqueness of the solution, by making use of the weak formulation. Furthermore, we use a numerical scheme based on finite differences to present numerical results.

\section{Introduction}

The theory of linear poroelasticity has been introduced and rigorously improved by many authors, among others, Biot [1-4], Bear and Bachmat [5], Bémer et al. [6], Barucq et al. [7], and Zenisek [8]. Nowadays, this theory leads to many applications in different disciplines, such as oil exploration, biological phenomena, medical sciences [9], and military applications [10].

In our work, we are interested in the study of fluidsaturated porous media, subject to a random disturbance when the phenomenon of consolidation is realized (see [11, 12]). The study of poroelastic properties or the problem of acoustic wave propagation in saturated porous media, for example, in the oil exploration, has been based on two approaches (see, e.g., $[9,10,13])$. The first focuses on microscopic laws; that is, the pore becomes an entire field of study and then derives the macroscopic laws that are involved across the porous media as a whole. This is where the homogenization techniques are used, by considering the fact that the microscopic structure is repeated periodically which leads to the periodicity of the solutions [14]. The second approach, the more classical, was due to Biot in the 50 s, where he suggested to ignore everything that happens at the microscopic level, to apply the continuum mechanics principles at the macroscopic level.

\section{The Model}

Let $\Omega$ be a porous medium and $\rho(x)$ the density; we consider the porous matrix (porous or skeleton) to be filled with a relatively incompressible viscous fluid which diffuses through. The small movements of both solid and liquid phases are verified, neglecting the speed of filtration. We write the coupled system of hyperbolic-parabolic type as

$$
\begin{aligned}
& \rho(x) \frac{\partial^{2} u}{\partial t^{2}}-\nabla\left(\lambda^{*}(x) \frac{\partial}{\partial t} \operatorname{div} u\right) \\
& \quad-\nabla((\lambda(x)+\mu(x)) \operatorname{div} u)-\operatorname{div}(\mu(x) \nabla u) \\
& \quad+\alpha \nabla p=f(t, x), \\
& c_{0}(x) \frac{\partial p}{\partial t}+\alpha \operatorname{div} \frac{\partial u}{\partial t}-\operatorname{div}(k(x) \nabla p)=h(t, x),
\end{aligned}
$$

where $u(t, x), p(t, x)$, and $f(t, x)$ are the velocity of the solid matrix, the fluid pressure, and the external forces acting on the macroscopic element, for all $x$ in $\Omega$ and all $t>0$, respectively, with $h(t, x)$ a source term. The parameters $\lambda(x)$ and $\mu(x)$ denote the expansion and the shear modulus, respectively, whereas the coefficients $\lambda^{*}(x), c_{0}(x)$, and $k(x)$ are positive such that $\lambda^{*}(x)$ is associated with the consolidation side effects and may cancel, $c_{0}(x) \geq 0$ is the coefficient that combines the porosity of the medium and 
the compressibility of the fluid-solid structure and $k(x)$ is the one which takes into account the permeability of the medium and the viscosity of the fluid, since it is a measure of flow obeying the Darcy law for a given pressure gradient. The constant $\alpha$ is a positive real number, representing the Biot-Willis constant which takes into account the effects of coupling the deformation and the pressure: it is actually a measure of the amount of fluid that can be placed in the porous matrix by increasing the pressure at constant volume.

\section{One-Dimensional Biot Model}

We are interested in the one-dimensional nonlinear model of the following form:

$$
\begin{aligned}
& \rho \partial_{t}^{2} u-\lambda^{*} \partial_{t} \partial_{x}^{2} u-(\lambda+2 \mu) \partial_{x}^{2} u-\mu^{*} \partial_{x}\left(\left|\partial_{x} u\right|^{q-2} \partial_{x} u\right) \\
& \quad+\alpha \partial_{x} p=f, \\
& c_{0} \partial_{t} p+\alpha \partial_{t} \partial_{x} u-k \partial_{x}^{2} p=h .
\end{aligned}
$$

The nonlinear term in the first equation of (2) is due to the local geometry of the medium, such as sudden changes in contact areas or occlusions of cracks, with $\mu^{*}(x) \geq 0$. Here $q \geq 0$ and $\lambda^{*}$ is a positive constant if $q \neq 2$ and vanishes when $q=2$. Besides the system physical parameters are assumed to be constant and independent of the space variable.

This system appears when we consider the particular case of a wave propagating in a single direction. In this case the displacement depends only on one variable denoted by $x$ and the scalar $u$ represents the component along the $x$-direction and the same for the pressure $p$.

Let us consider $\Omega=[a, b], a, b \in \mathbb{R}, a<b$, to be the saturated porous medium which occurs in the propagation of the wave and let $Q$ be the cylinder $Q=[0, T] \times \Omega$.

When $\rho=0$, the system is transformed into a quasistatic system as follows:

$$
\begin{aligned}
& -\lambda^{*} \partial_{t} \partial_{x}^{2} u-(\lambda+2 \mu) \partial_{x}^{2} u-\mu^{*} \partial_{x}\left(\left|\partial_{x} u\right|^{q-2} \partial_{x} u\right) \\
& \quad+\alpha \partial_{x} p=f \\
& c_{0} \partial_{t} p+\alpha \partial_{t} \partial_{x} u-k \partial_{x}^{2} p=h
\end{aligned}
$$

with the initial conditions

$$
(u(0, x), p(0, x))=\left(u_{0}(x), p_{0}(x)\right) .
$$

In addition, when $\rho>0$, we can write

$$
\partial u(0, x)=u_{1}(x)
$$

and the homogeneous Dirichlet boundary conditions

$$
u(t, a)=u(t, b)=p(t, a)=p(t, b)=0 .
$$

Note that $\lambda, \mu, \alpha$, and $c_{0}$ are strictly positive constants.

3.1. Galerkin Method. The Galerkin method is used to prove the existence of the discrete solution. We consider a family of vector spaces $\left(V_{m}\right)_{m \in \mathbb{N}^{*}}$ that approaches an infinite dimensional Hilbert space $V$ satisfying the following:

(i) $\left(V_{m}\right)_{m \in \mathbb{N}^{*}} \subset V$;

(ii) $V_{m} \rightarrow V$ when $m \rightarrow+\infty$ in the sense that there exists a dense subspace $v$ of $V$, such that, for all $v \in V$, we can find a sequence $\left(v_{m}\right)_{m \in \mathbb{N}^{*}}$ satisfying: for all $m$, $v_{m} \in V_{m}$, and $v_{m} \rightarrow v$ in $V$ when $m \rightarrow+\infty$.

These approximation spaces are generated using a family $\left(w_{j}\right)_{j \in \mathbb{N}^{*}}$ for $V$ such that, for $m \in \mathbb{N}^{*}, V_{m}=$ $\operatorname{Vect}\left\{w_{1}, w_{2}, \ldots, w_{m}\right\}$. According to the choice of this family, we can construct solutions to problems that can be more or less regular.

In order to have sufficient regularity for the nonlinear term treatment, we make use of the eigenfunctions of the Laplace operator in $L^{2}(\Omega)$. First let us set the following results.

\subsection{Known Results for Laplace’s Operator}

Proposition 1. There exists a sequence $\left(w_{j}\right)_{j \in \mathbb{N}^{*}}$ such that

$$
\begin{gathered}
\forall j \in \mathbb{N}^{*}, \quad w_{j} \in H_{0}^{1}(\Omega) \cap H^{2}(\Omega) \\
-\Delta w_{j}=\lambda_{j} w_{j}
\end{gathered}
$$

with $w_{j} \neq 0$. The set $\left(w_{j}\right)_{j \in \mathbb{N}^{*}}$ is a Hilbertian basis for $L^{2}(\Omega)$ and the space of the finite linear combinations of $w_{j}$ is dense in $H_{0}^{1}(\Omega)$ and in $H_{0}^{1}(\Omega) \cap H^{2}(\Omega)$.

Proposition 2. Let $V_{m}=\operatorname{Vect}\left\{w_{1}, w_{2}, \ldots, w_{m}\right\}$ where $\left(w_{j}\right)_{j \in \mathbb{N}^{*}}$ is defined by (7) and let $P_{m}$ be the projection onto $V_{m}$ defined on $\left(H_{0}^{1}(\Omega) \cap H^{2}(\Omega)\right)^{\prime}$ by

$$
P_{m}(v)=\sum_{i=1}^{m}\left\langle v, w_{i}\right\rangle w_{i},
$$

where $\langle\cdot, \cdot\rangle$ denotes the duality product between $H_{0}^{1}(\Omega) \cap$ $H^{2}(\Omega)$ and its dual. Then the restriction of $P_{m}$ to $L_{2}(\Omega)$ on $V_{m}$ is $P_{m} \in \mathscr{L}\left(L_{2}(\Omega)\right)$ with $\left\|P_{m}\right\|_{\mathscr{L}\left(L_{2}(\Omega)\right)}=1$. In addition, the following properties

$$
\begin{aligned}
& P_{m} \in \mathscr{L}\left(H_{0}^{1}(\Omega) \cap H^{2}(\Omega)\right), \\
& P_{m} \in \mathscr{L}\left(\left(H_{0}^{1}(\Omega) \cap H^{2}(\Omega)\right)^{\prime}\right)
\end{aligned}
$$

are satisfied and the norms $\left\|P_{m}\right\|_{\mathscr{L}\left(\left(H_{0}^{1}(\Omega) \cap H^{2}(\Omega)\right)^{\prime}\right)}$ and $\left\|P_{m}\right\|_{\mathscr{L}\left(H_{0}^{1}(\Omega) \cap H^{2}(\Omega)\right)}$ are independent of $m$.

\section{One-Dimensional Nonlinear Stochastic Biot Model}

Here we are interested in studying the Biot model in the presence of a stochastic perturbation. The idea is to develop a mathematical analysis of the above equations with a stochastic perturbation. This is based on analyzing the problem in a weak form by making use of appropriate functional spaces. 
4.1. Weak Formulation. We study the model of consolidation in the case where $\rho \geq 0$ and $q \geq 2$ by using the weak formulation.

Find $(u, p) \in L^{\infty}\left([0, T] ; W_{0}^{1, q}(\Omega)\right) \times L^{\infty}\left([0, T] ; H_{0}^{1}(\Omega)\right)$ such that $u \in L^{2}\left([0, T] ; W^{2}(\Omega)\right), \partial_{t} u \in L^{2}\left([0, T] ; W_{0}^{1}(\Omega)\right)$, $\partial_{t} p \in L^{2}\left([0, T] ; L^{2}(\Omega)\right)$, and $\rho \partial_{t}^{2} u \in L^{2}\left([0, T] ; W^{-1, q^{*}}(\Omega)\right)$, satisfying

$$
\begin{gathered}
\rho\left\langle\partial_{t}^{2} u, v\right\rangle_{W^{-1, q^{*}}(\Omega), W_{0}^{1, q}(\Omega)}+\lambda^{*} \int_{\Omega} \partial_{t} \partial_{x} u \partial_{x} v d x \\
-\alpha \int_{\Omega} p \partial_{x} v d x+(\lambda+2 \mu) \int_{\Omega} \partial_{x} u \partial_{x} v d x \\
+\mu^{*} \int_{\Omega}\left|\partial_{x} u\right|^{q-2} \partial_{x} u \partial_{x} v d x=\int_{\Omega} f v d x+\int_{\Omega} v d G \\
c_{0} \int_{\Omega} \partial_{t} p r d x+\alpha \int_{\Omega} \partial_{t} \partial_{x} u r d x \\
+k \int_{\Omega} \partial_{x} p \partial_{x} r d x=\int_{\Omega} h r d x \\
(u(0, x), p(0, x))=\left(u_{0}(x), p_{0}(x)\right) \\
\partial u(0, x)=u_{1}(x)
\end{gathered}
$$

for almost every $t$ in $] 0, T\left[\right.$ and $\forall(v, r) \in W_{0}^{1, q}(\Omega) \times H_{0}^{1}(\Omega)$. Here $q^{*}$ is real and is defined by the relation $1 / q+1 / q^{*}=1$. It is assumed that the initial conditions $\left(u_{0}, p_{0}\right)$ and $u_{1}$ (when $\rho>0)$ are in $H_{0}^{1}(\Omega) \cap H^{2}(\Omega) \times H_{0}^{1}(\Omega)$ and $L^{2}(\Omega)$, respectively, and the source terms belong to $L^{2}\left([0, T] ; L^{2}(\Omega)\right)$. We are also supposed to have a major regularity for the disturbance $G$, so that the resolution of the stochastic equation is reduced for each element $\omega$ of the probability space $\Omega$, to a deterministic equation. Hence $G$ is a continuous stochastic process with values in $\Omega$ (i.e., continuous trajectory of the disturbance) and defined on a probability space $(\Omega, F, P)$. Any solution of the variational formulation (10) is called the solution of the nonlinear stochastic consolidation Biot model. This solution is obtained by solving the equations in (10) for each $\omega \in \Omega$.

Theorem 3. Let q be real such that $q \geq 2, u_{0} \in H_{0}^{1}(\Omega) \cap H^{2}(\Omega)$, $p_{0} \in H_{0}^{1}(\Omega), u_{1} \in L^{2}(\Omega)$, and $f$ and $h \in L^{2}\left([0, T] ; L^{2}(\Omega)\right)$. Let $G$ be a stochastic process defined in $\Omega$. There is a single pair of random variables $(u, p)$ such that $u \in L^{\infty}\left([0, T] ; W^{1, \infty}(\Omega)\right)$ and $p \in L^{\infty}\left([0, T] ; H_{0}^{1}(\Omega)\right)$, satisfying the system of $(10)$.

4.2. Existence of the Solution. In this section, we propose to formulate the equations whose solutions constitute the Faedo-Galerkin approximation type of our problem, in $H_{0}^{1}(\Omega) \cap H^{2}(\Omega)$ (for more details on the Faedo-Galerkin approximations the reader is referred to $[15,17]$ and references therein). A solution is constructed as the limit of a sequence of approximate solutions denoted by $\left(u_{m}, p_{m}\right)_{m \in \mathbb{N}^{*}}$. This sequence $\left(u_{m}, p_{m}\right)_{m \in \mathbb{N}^{*}}$ is defined from $] 0, T\left[\right.$ in $V_{m} \times V_{m}$ by

$$
\begin{aligned}
& u_{m}(t)=\sum_{j=1}^{m} u_{j m}(t) w_{j}, \\
& p_{m}(t)=\sum_{j=1}^{m} p_{j m}(t) w_{j},
\end{aligned}
$$

where $\left(w_{j}\right)_{j \in \mathbb{N}^{*}}$ is the sequence defined in Proposition 1 of Section 3.2. To each integer $m \in \mathbb{N}^{*}$, we associate a new discrete unknown by the use of the sequence $\left(u_{j m(t)}, p_{j m(t)}\right)_{1<j<m}$ that is defined by solving the differential system

$$
\begin{aligned}
& \rho \int_{\Omega} \partial_{t}^{2} u_{m} w_{j} d x+\lambda^{*} \int_{\Omega} \partial_{t} \partial_{x} u_{m} \partial_{x} w_{j} d x \\
& \quad+(\lambda+2 \mu) \int_{\Omega} \partial_{x} u_{m} \partial_{x} w_{j} d x \\
& \quad+\mu^{*} \int_{\Omega}\left|\partial_{x}\right|^{q-2} u_{m} \partial_{x} u_{m} \partial_{x} w_{j} d x \\
& \quad-\alpha \int_{\Omega} p_{m} \partial_{x} w_{j} d x=\int_{\Omega} f w_{j} d x+\int_{\Omega} w_{j} d G_{m} p \cdot s \\
& c_{0} \int_{\Omega} \partial_{t} p_{m} w_{j} d x+\alpha \int_{\Omega} \partial_{t} \partial_{x} u_{m} w_{j} d x \\
& \quad+k \int_{\Omega} \partial_{x} p_{m} \partial_{x} w_{j} d x=\int_{\Omega} h w_{j} d x,
\end{aligned}
$$

for all $1 \leq j \leq m$, with initial conditions

$$
\begin{aligned}
&\left(u_{m}(0), p_{m}(0)\right)=\left(u_{0 m}, p_{0 m}\right) \in V_{m} \times V_{m} \text { such that } \\
& u_{0 m} \longrightarrow u_{0} \quad \text { in } H_{0}^{1}(\Omega) \cap H^{2}(\Omega), \\
& p_{0 m} \longrightarrow p_{0} \quad \text { in } H_{0}^{1}(\Omega) \\
& \rho \partial_{t} u_{m}(0)=\rho u_{1 m} \in V_{m} \text { such that } \\
& p_{1 m} \longrightarrow p_{1} \quad \text { in } L^{2}(\Omega)
\end{aligned}
$$

where $u(\omega, t) \in V_{m}, \forall t \in[0, T]$ p.s.

$G_{m}$ is a continuous stochastic process with values in $V_{m}$ and defined on a probability space $(\Omega, F, P)$. The solution of (12) is obtained by solving the equations for each fixed $\omega \in \Omega$. Therefore we consider the following deterministic equations:

$$
\begin{gathered}
\rho \int_{\Omega} \partial_{t}^{2} u_{m} w_{j} d x+\lambda^{*} \int_{\Omega} \partial_{t} \partial_{x} u_{m} \partial_{x} w_{j} d x \\
+(\lambda+2 \mu) \int_{\Omega} \partial_{x} u_{m} \partial_{x} w_{j} d x \\
+\mu^{*} \int_{\Omega}\left|\partial_{x}\right|^{q-2} \partial_{x} u_{m} \partial_{x} w_{j} d x \\
-\alpha \int_{\Omega} p_{m} \partial_{x} w_{j} d x \\
=\int_{\Omega} f w_{j} d x+\int_{\Omega} G_{m} w_{j} d x \\
c_{0} \int_{\Omega} \partial_{t} p_{m} w_{j} d x+\alpha \int_{\Omega} \partial_{t} \partial_{x} u_{m} w_{j} d x \\
+k \int_{\Omega} \partial_{x} p_{m} \partial_{x} w_{j} d x=\int_{\Omega} h w_{j} d x
\end{gathered}
$$


for all $1 \leq j \leq m$, with initial conditions

$$
\begin{gathered}
\left(u_{m}(0), p_{m}(0)\right) \\
=\left(u_{0 m}, p_{0 m}\right) \in V_{m} \times V_{m} \text { such that } \\
u_{0 m} \longrightarrow u_{0} \quad \text { in } H_{0}^{1}(\Omega) \cap H^{2}(\Omega), \\
\quad p_{0 m} \longrightarrow p_{0} \quad \text { in } H_{0}^{1}(\Omega) \\
\rho \partial_{t} u_{m}(0)=\rho u_{1 m} \in V_{m} \text { such that } \\
u_{1 m} \longrightarrow u_{1} \quad \text { in } L^{2}(\Omega)
\end{gathered}
$$

with $u(t) \in V_{m}, \forall t \in[0, T]$, and $G_{m}$ is a continuous function with values in $V_{m}$.

Theorem 4. The existence of sequences $\left(u_{0 m}\right)_{m \in \mathbb{N}^{*}},\left(u_{1 m}\right)_{m \in \mathbb{N}^{*}}$, and $\left(p_{0 m}\right)_{m \in \mathbb{N}^{*}}$ satisfying the properties (13 bis) is a consequence of Propositions 1 and 2 in Section 3.2. Problem ((12 bis)-(13 bis)) satisfies the Cauchy-Lipschitz conditions and, from the nonlinear differential equations theory, ((12 bis) (13 bis)) admits a unique maximal solution $\left(u_{m}, p_{m}\right)_{m \in \mathbb{N}^{*}}$ in $H^{1}\left(\left[0, T_{m}\right] ; H_{0}^{1}(\Omega) \cap H^{2}(\Omega)\right) \times H^{1}\left(\left[0, T_{m}\right]\right.$; $\left.H_{0}^{1}(\Omega)\right), T_{m}>0$, such that $\rho u_{m} \in H^{2}\left(\left[0, T_{m}\right] ; H_{0}^{1}(\Omega) \cap\right.$ $\left.H^{2}(\Omega)\right)$.

The idea is to show that there exists $\left(u_{m}, p_{m}\right)_{m \in \mathbb{N}^{*}}$ that converges to problem ((12 bis)-(13 bis)) solution $(u, p)$. It is sufficient to extract a converging sequence $\left(u_{m}, p_{m}\right)_{m \in \mathbb{N}^{*}}$, where its existence is satisfied by a priori estimates which prove that the sequence is bounded in suitable functional spaces from the bounded differential equation solution's principle. The time $T_{m}$ for which solutions exist is equal to the initially given time $T$.

Lemma 5. The sequence $\left(u_{m}, p_{m}\right)_{m \in \mathbb{N}^{*}}$ of the solutions of problem ((12 bis)-(13 bis)) satisfies the following properties:

(i) $\left(\rho \partial_{t}^{2} u_{m}\right)_{m \in \mathbb{N}^{*}}$ is bounded in $L^{2}\left([0, T] ;\left(H_{0}^{1}(\Omega) \cap\right.\right.$ $\left.\left.H^{2}(\Omega)^{\prime}\right)\right)$,

(ii) $\left(\partial_{t} u_{m}\right)_{m \in \mathbb{N}^{*}}$ is bounded in $L^{2}\left([0, T] ; H_{0}^{1}(\Omega)\right)$,

(iii) $\left(\sqrt{\rho} \partial_{t} u_{m}\right)_{m \in \mathbb{N}^{*}}$ is bounded in $L^{\infty}\left([0, T] ; L^{2}(\Omega)\right)$,

(iv) $\left(u_{m}\right)_{m \in \mathbb{N}^{*}}$ is bounded in $L^{\infty}\left([0, T] ; W^{1, q}(\Omega)\right)$,

(v) $\left(\partial_{t} p_{m}\right)_{m \in \mathbb{N}^{*}}$ is bounded in $L^{2}\left([0, T] ; L^{2}(\Omega)\right)$,

(vi) $\left(p_{m}\right)_{m \in \mathbb{N}^{*}}$ is bounded in $L^{\infty}\left([0, T] ; H_{0}^{1}(\Omega)\right)$.

Each estimate is independent of the physical parameter $\rho$ but depends only on $\left(u_{0}, p_{0}\right)$ and $u_{1}$ from $\left\|u_{0}\right\|_{W^{1, q}(\Omega)}$, $\left\|p_{0}\right\|_{H^{1}(\Omega)}$, and $\left\|u_{1}\right\|_{L^{2}(\Omega)}$.

Lemma 6. Let $\left(u_{m}, p_{m}\right)_{m \in \mathbb{N}^{*}}$ be the sequence of the solutions of ((12 bis)-(13 bis $))$. Then

(i) $\left(\partial_{x}^{2} u_{m}\right)_{m \in \mathbb{N}^{*}}$ is bounded in $L^{\infty}\left(0, T ; L^{2}(\Omega)\right)$,

(ii) $\left(u_{m}\right)_{m \in \mathbb{N}^{*}}$ is bounded in $L^{\infty}\left(0, T ; W^{1, q}(\Omega)\right)$.

For the proof of Lemma 6, we use the same as arguments as [7].

For the proof of Theorem 3, we proceed as follows.
From Theorem 4 we have, for almost every $\omega$, problem ((12 bis), (13 bis)) with $G_{m}=G_{m}(\omega)$ having one and only one solution: $(u, p)=(u(\omega), p(\omega)) \in C\left(0, T ; V_{m}\right) \times C^{1}\left(0, T ; V_{m}\right)$, satisfying ((12), (13)). It is sufficient to prove that $G_{m} \in$ $C\left(0, T ; V_{m}\right) \rightarrow(u, p)$ defined by the unique solution in Theorem 4 is measurable. Let $G_{m}$ and $\overline{G_{m}}$ be two elements of $C\left(0, T ; V_{m}\right)$ and let $(u, p)$ be the solution of ((12 bis), (13 bis)) corresponding to $G_{m}$ and $\overline{G_{m}}$, respectively. Using the uniqueness of the solution we have

$$
\begin{gathered}
v=u-\bar{u}=0 \\
\psi=p-\bar{p}=0 .
\end{gathered}
$$

That is to say $(u-\bar{u}) \rightarrow 0$ in $L^{\infty}\left(0, T ; W_{0}^{1, q}(\Omega)\right)$ and $(p-\bar{p}) \rightarrow$ 0 in $L^{\infty}\left(0, T ; H_{0}^{1}(\Omega)\right)$.

This proves that the mapping: $G_{m} \rightarrow(u, p)$ is continuous and, from the compacity, $G_{m}$ is measurable in the considered topology.

\section{Numerical Results}

5.1. Discretisation and Stability of the Scheme. We consider (2), taking $q=2$ and $f=f+d W$ :

$$
\begin{gathered}
-\lambda^{*} \partial_{t} \partial_{x}^{2} u-\left(\lambda+2 \mu+\mu^{*}\right) \partial_{x}^{2} u+\alpha \partial_{x} p=f+d W \\
c_{0} \partial_{t} p+\alpha \partial_{t} \partial_{x} u-k \partial_{x}^{2} p=h .
\end{gathered}
$$

We use the Euler scheme for the time discretisation, the central finite differences for the space variable, and

$$
d W=W(n+1)-W(n)=\sqrt{n} \operatorname{rand} n
$$

to obtain

$$
\begin{aligned}
U_{j+1}^{n+1}= & a_{1} U_{j}^{n+1}+U_{j-1}^{n+1}+a_{2} U_{j+1}^{n}+a_{3} U_{j}^{n} \\
& +a_{4} U_{j-1}^{n} a_{5} U_{j}^{n-1} a_{6}\left(P_{j+1}^{n}-P_{j}^{n}\right)+a_{7} \\
P_{j}^{n+1}= & b_{1}\left(P_{j+1}^{n}+P_{j-1}^{n}\right)+b_{2} P_{j}^{n} \\
& +b_{3}\left(U_{j-1}^{n+1}-U_{j}^{n+1}-U_{j+1}^{n}+U_{j}^{n}\right)+h^{*} d t
\end{aligned}
$$

with $a_{1}=\left(1 / \rho^{*} d t+2 /(d x)^{2}\right) ; a_{2}=\left(d t A^{*} / \rho^{*}(d x)^{2}-1 /(d x)^{2}\right)$; $a_{3}=\left(2 / \rho^{*} d t+2 /(d x)^{2}-2 d t A^{*} / \rho^{*}(d x)^{2}\right) ; a_{4}=\left(d t A^{*} /\right.$ $\left.\rho^{*}(d x)^{2}-1 /(d x)^{2}\right) ; a_{5}=\left(-1 / \rho^{*} d t\right) ; a_{6}=\left(\alpha^{*} d t / \rho^{*} d x\right)$, $a_{7}=\left(B^{*} d t / \rho^{*}\right), b_{1}=d t k^{*} /(d x)^{2} ; b_{2}=\left(1-2 d t k^{*} /(d x)^{2}\right)$, $b_{3}=\left(-c^{*} / d x\right), A=\lambda+2 \mu+\mu^{*} ; B=f+d W ; c^{*}=\alpha / c_{0}$; $k^{*}=k / c_{0} ; h^{*}=h / c_{0} ; \rho^{*}=\lambda^{*} / \rho ; A^{*}=A / \rho ; \alpha^{*}=\alpha / \rho$; $B^{*}=B / \rho$.

Using the Fourier stability analysis, we write

$$
\frac{2}{(d x)^{2}}+\frac{1}{\rho^{*} d t}<1
$$




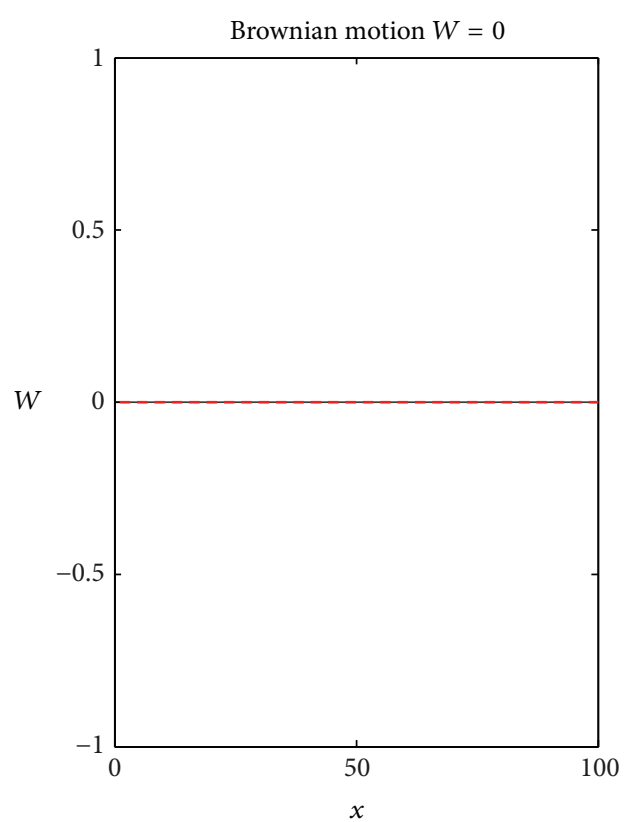

(a)

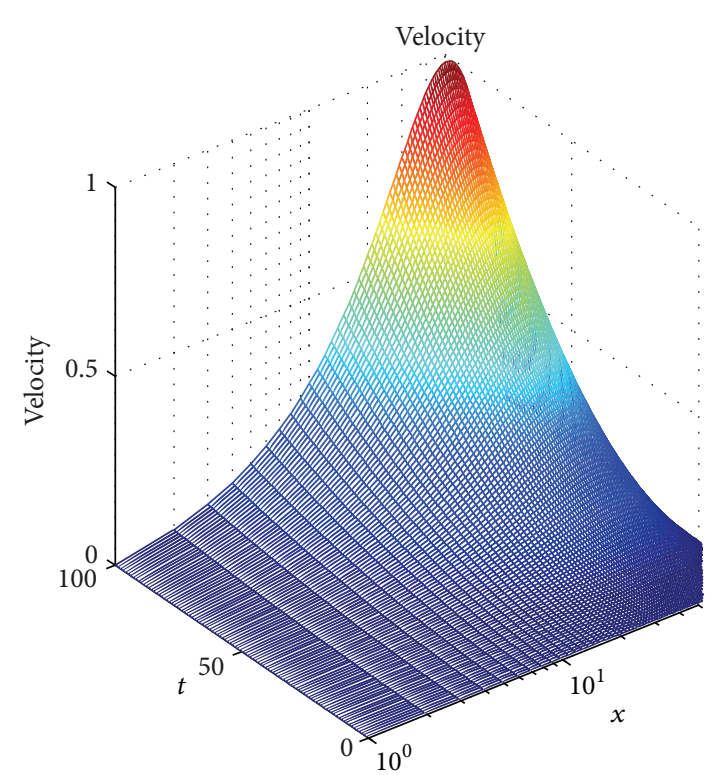

(b)

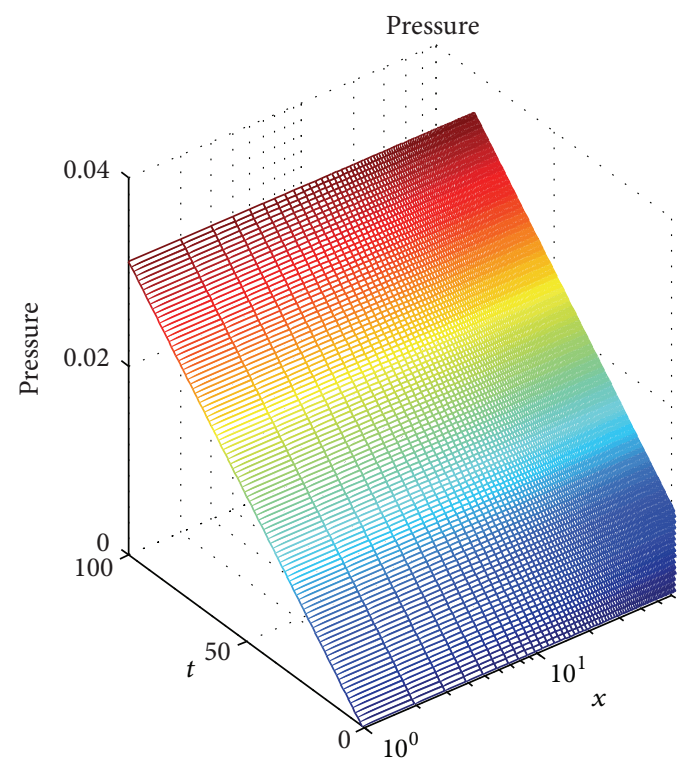

(c)

Figure 1: A null Brownian motion and the associated velocity and pressure.

This is the so-called CFL condition, which is the stability condition leading us to the right choice of the discretisation time and space steps.

5.2. Numerical Results and Comments. In Figures 1-3, we present the Brownian motion, the associated velocities, and pressures for different space and time steps as solutions of (15) using the finite difference scheme considered in Section 5.1. The numerical simulations were carried out using MATLAB with the parameters set as $\rho=0.04, \lambda=0.02, \lambda^{*}=0.03$, $\mu=0.03, \mu^{*}=0.07, c_{0}=0.05, \alpha=0.01$, and $k=0.03$. The data are set as $f=10$ and $h=2.0$.
5.2.1. Comments on the Numerical Results. According to the numerical experimentations, in Figure 1, we present results for the deterministic case where the Brownian motion $W$ is equal to 0. As shown in Figures 2 and 3, it is clear that the velocity and the pressure of the fluid behave randomly because of the stochastic part in (10). We also noticed that we experimented no unstability behavior as long as we respect the CFL condition (the stability condition) that is deducted from the stability analysis of the finite difference scheme considered (see Figure 3). To show this numerical instability behavior, we present numerical results for a condition equal to twice the CFL condition (see Figure 2). 
6

Abstract and Applied Analysis

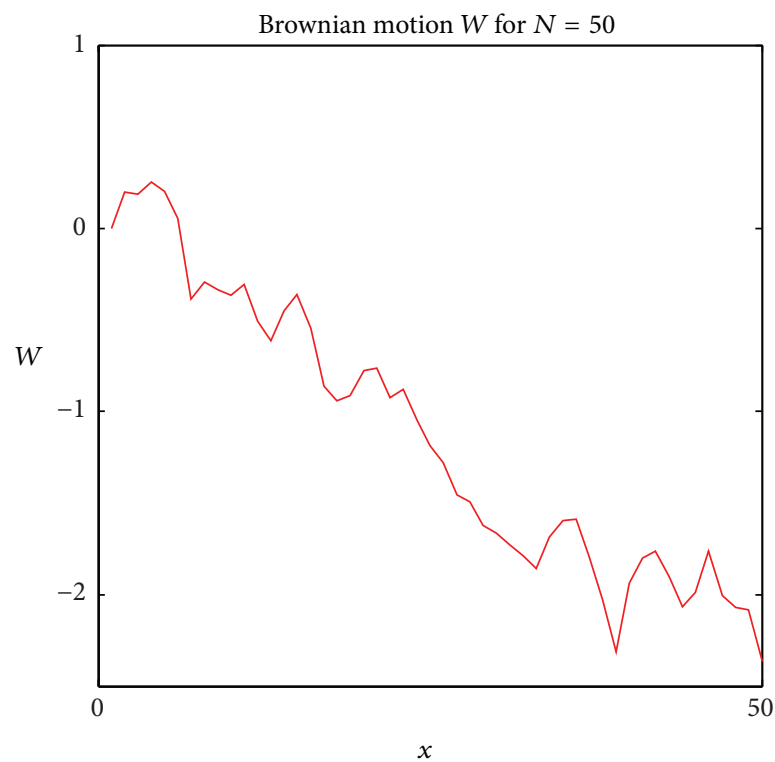

(a)

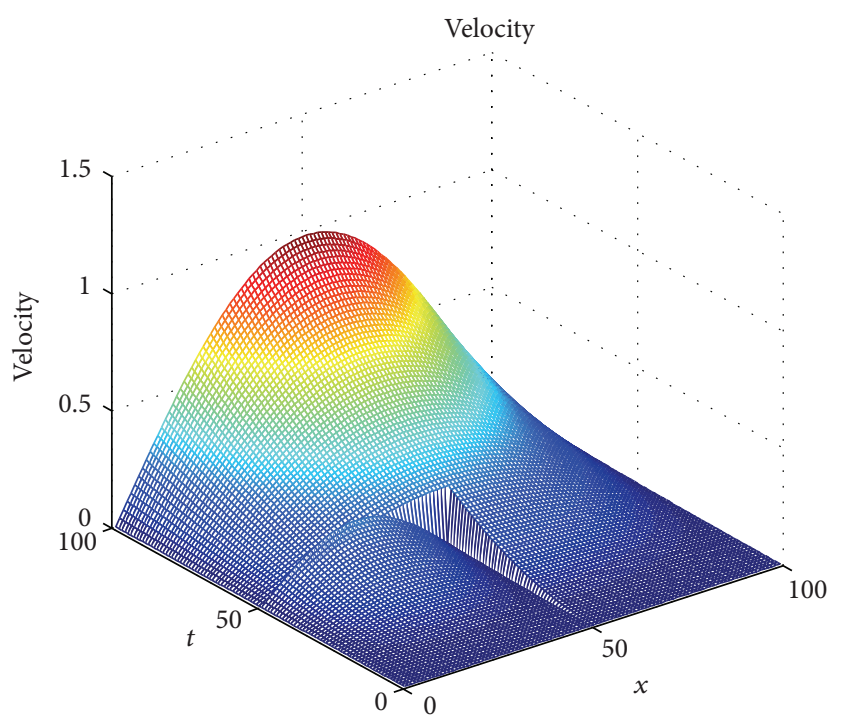

(b)

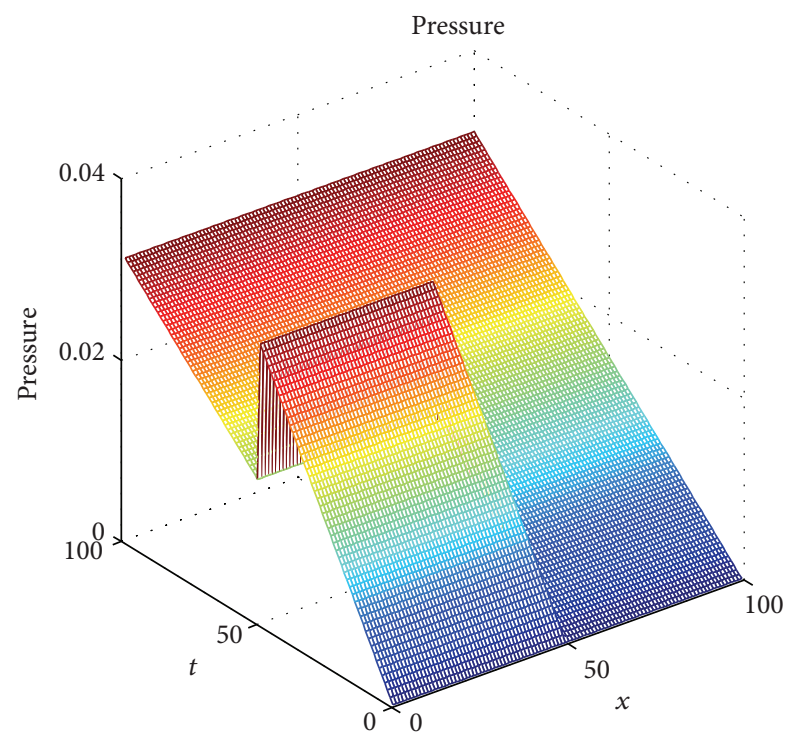

(c)

FiguRE 2: The Brownian, the associated velocity and pressure, and instable case.

5.3. Conclusion. We solved a system of two partial differentaal equations (IDEs) in a bounded domain, modeling the velocity and pressure; the first equation has a nonlinear part and another random part. A weak solution has been found in the space $H_{0}^{1}(\Omega) \cap H^{2}(\Omega)$ by the principle of the resolution of a stochastic differential equation (SDE) trajectory path. The velocity and the pressure were approached numerically by the Euler method for the time and the central finite difference for the space variable, where the time and space discretisation steps were chosen according to the numerical stability condition (CFL). The numerical results are resumed in Figures 1-3 and commented on above. It has to be pointed out that this model is quite important and can be used in many applications, for example, in image processing, and for this purpose a study on a comparison of this model with the one in [16] is under consideration.

Conflict of Interests

The author declares that there is no conflict of interests regarding the publication of this paper.

Acknowledgments

This work was supported by the Algerian Research National Project PNR977/23/2011-2013. The author is grateful to Professors H. F. Yashima and F. Z. Nouri for fruitful discussions, 


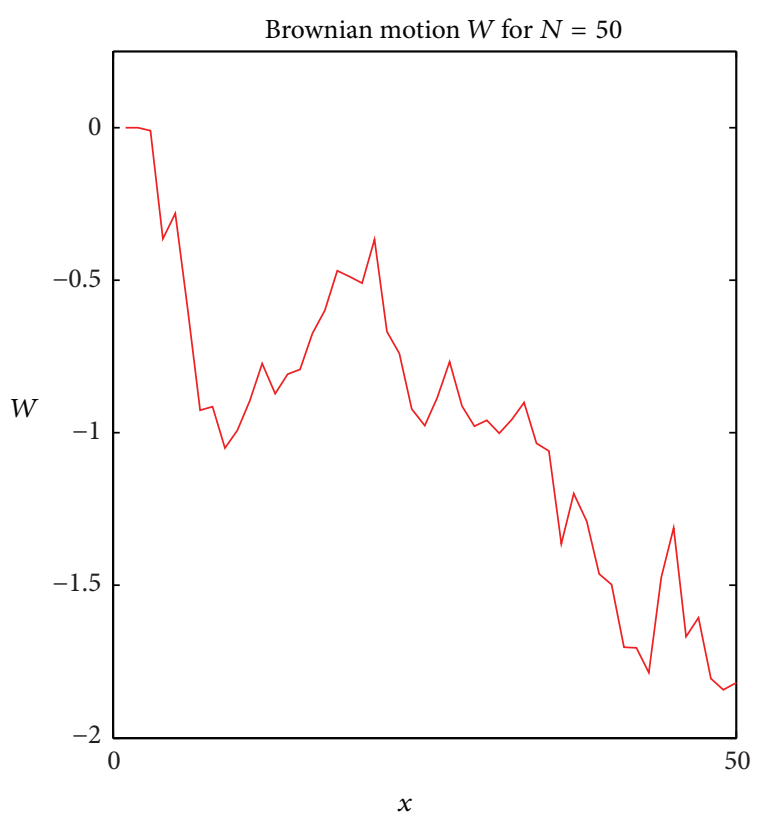

(a)

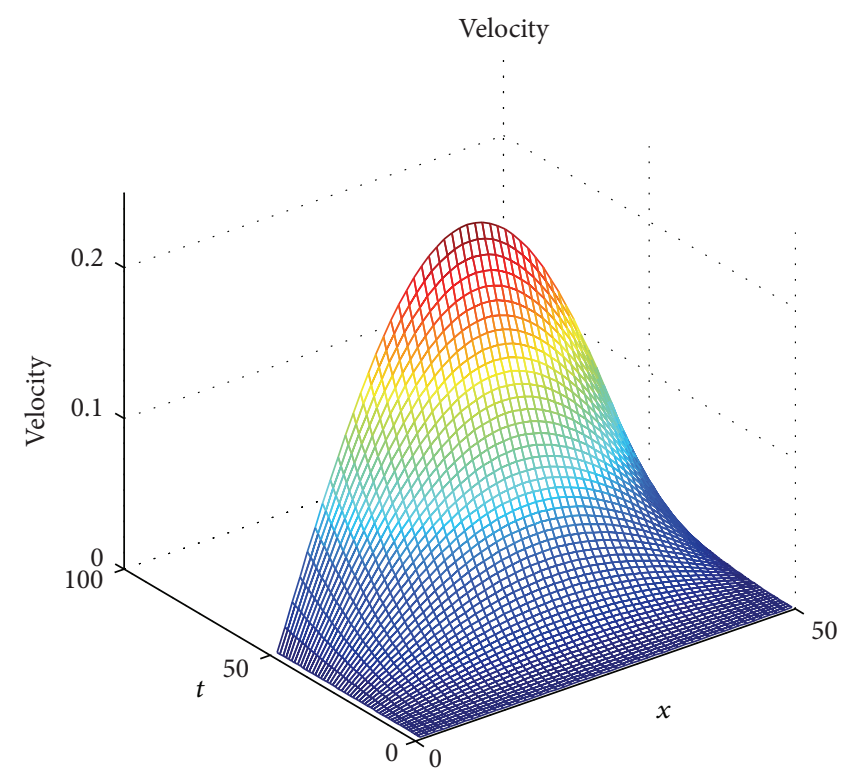

(b)

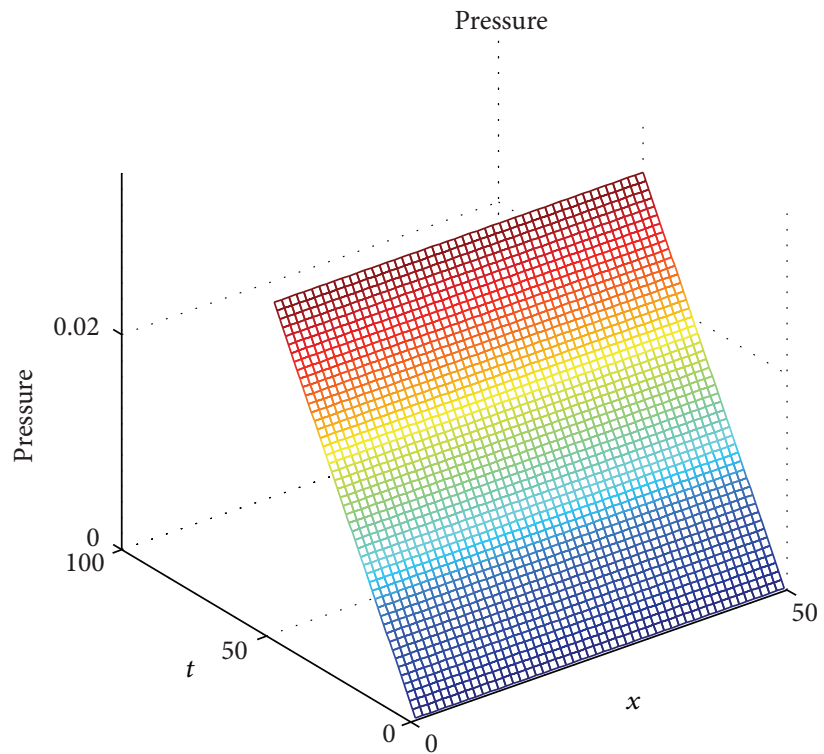

(c)

FIgURE 3: The Brownian, the associated velocity and pressure, and stable case.

recommendations, and suggestions that made the accomplishment of this work possible.

\section{References}

[1] M. A. Biot, "Le problème de la consolidation de Matières Argileuses sous une charge," Annales de la Société Scientifique de Bruxelles B, vol. 55, pp. 110-113, 1935.

[2] M. A. Biot, "General theory of three-dimensional consolidation," Journal of Applied Physics, vol. 12, no. 2, pp. 155-164, 1941.

[3] M. A. Biot, "Theory of elasticity and consolidation for a porous anisotropic solid," Journal of Applied Physics, vol. 26, pp. 182$185,1955$.
[4] M. A. Biot, "Theory of deformation of a porous viscoelastic anisotropic solid," Journal of Applied Physics, vol. 27, pp. 459$467,1956$.

[5] J. Bear and Y. Bachmat, Introduction of Modeling of Transport Phenomena in Porous Media, Kluwer Academic Publishers, Dordrecht, The Netherlands, 1991.

[6] E. Bémer, M. Boutéca, O. Vincké, N. Hoteit, and O. Ozanam, "Poromechanics: from linear to nonlinear poroelasticity and poroviscoelasticity," Oil and Gas Science and Technology-Revue de l'IFP, vol. 56, no. 6, pp. 531-544, 2001.

[7] H. Barucq, M. Madaune-Tort, and P. Saint-Macary, "Some existence-uniqueness results for a class of one-dimensional 
nonlinear Biot models," Nonlinear Analysis: Theory, Methods \& Applications, vol. 61, no. 4, pp. 591-612, 2005.

[8] A. Zenisek, "The existence and uniqueness theorem in Biot's consolidation theory," Aplikace Matematiky, vol. 29, pp. 194-211, 1984.

[9] A. Hosokawa and T. Otani, "Ultrasonic wave propagation in bovine cancellous bone," Journal of the Acoustical Society of America, vol. 101, no. 1, pp. 558-562, 1997.

[10] Y. Q. Zeng and Q. H. Liu, "Acoustic detection of buried objects in 3-D fluid saturated porous media: numerical modeling," IEEE Transactions on Geoscience and Remote Sensing, vol. 39, no. 6, pp. 1165-1173, 2001.

[11] J. L. Auriault, "Sur la rhélogie d'un milieu poreux saturé consolidant," Archive of Journal of Mechanics, vol. 27, no. 2, pp. 363-370, 1975.

[12] J. L. Auriault and E. Sanchez-Palencia, "Etude du comportement macroscopique d.un milieu Poreux Saturé Deformable," Archive of Journal of Mechanics, vol. 5, 16, pp. 575-603, 1977.

[13] T. Bourbé, O. Coussy, and B. Zinszner, Acoustique des Milieux Poreux, Publications de l'Institut Français du Pétrole, Collection 'Science et Technique du Petrole', Technip, Paris, France, 1998.

[14] H. Brezis, Analyse Fonctionnelle, Masson, Paris, France, 1983.

[15] M. L. Hadji, "Etude de l'équation de Navier-Stokes stochastique non homogène," Revue Synthèse, no. 27, pp. 15-21, 2013.

[16] M. L. Hadji, M. Maouni, and F. Z. Nouri, "A wavelet inpainting by a tixotrop model," in Proceedings of the 14th International conference on Information Visualisation, IV, pp. 559-563, IEEE Computer Society, London, UK, July 2010.

[17] H. F. Yashima, Equations de Navier-Stokes stochastiques non homogènes et applications [Ph.D. thesis], Scuola Normale Superiore, Pisa, Italy, 1992. 


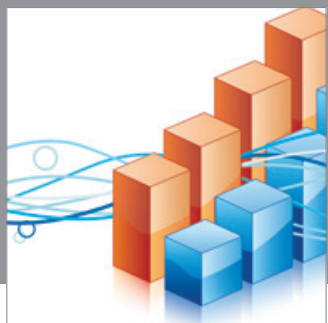

Advances in

Operations Research

mansans

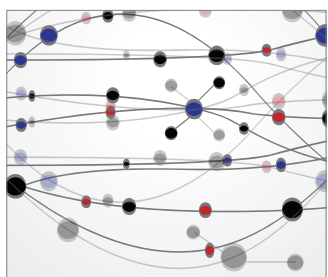

The Scientific World Journal
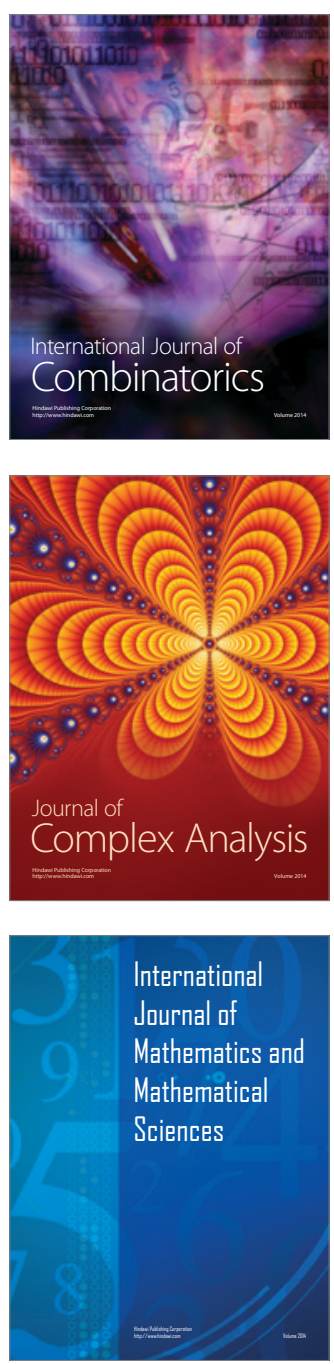
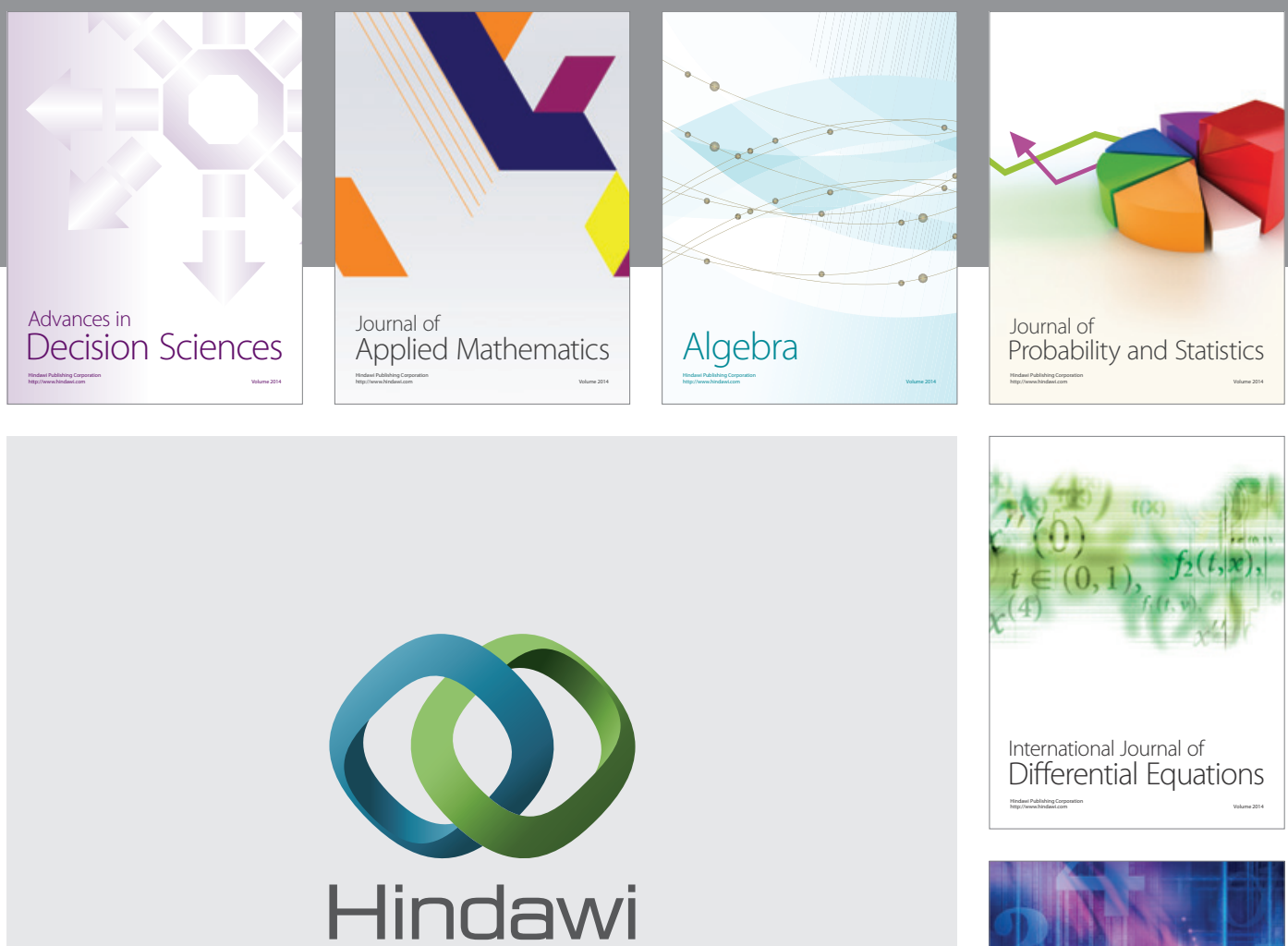

Submit your manuscripts at http://www.hindawi.com
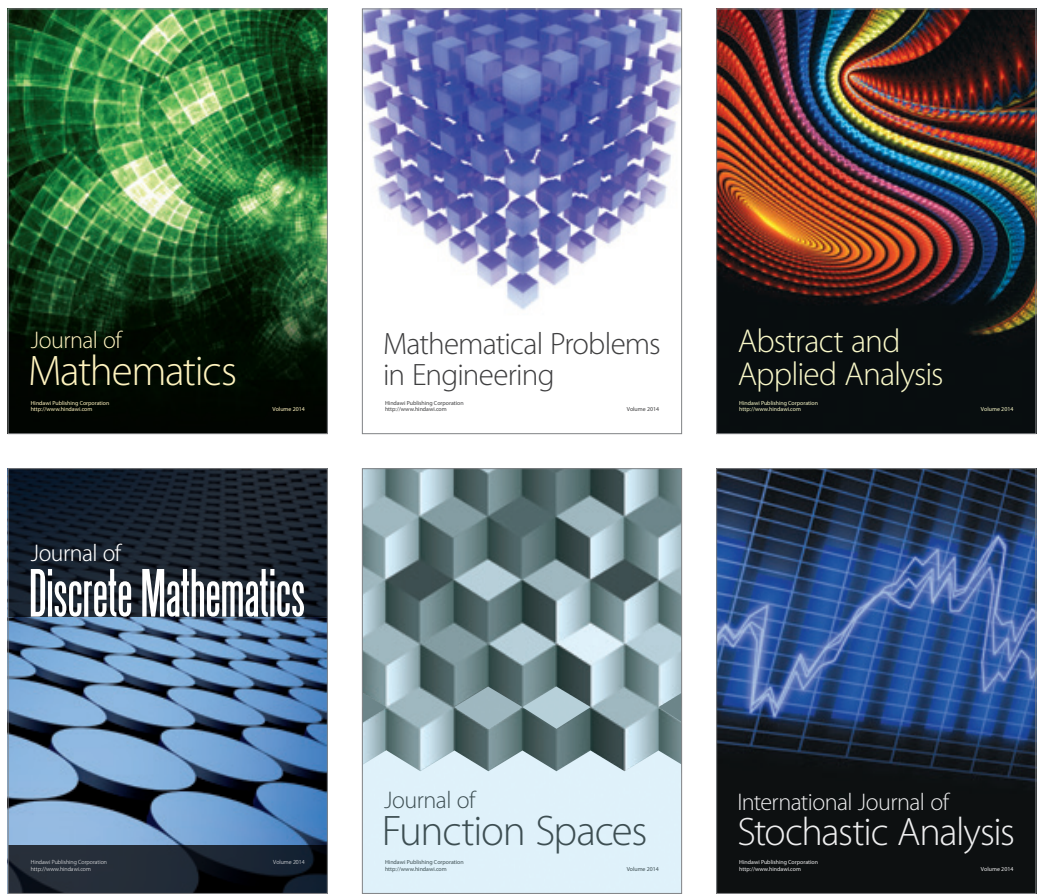

Journal of

Function Spaces

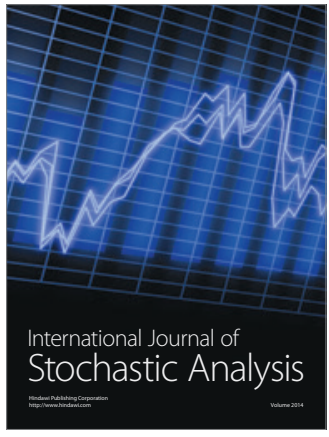

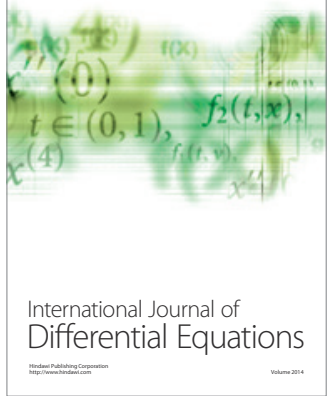
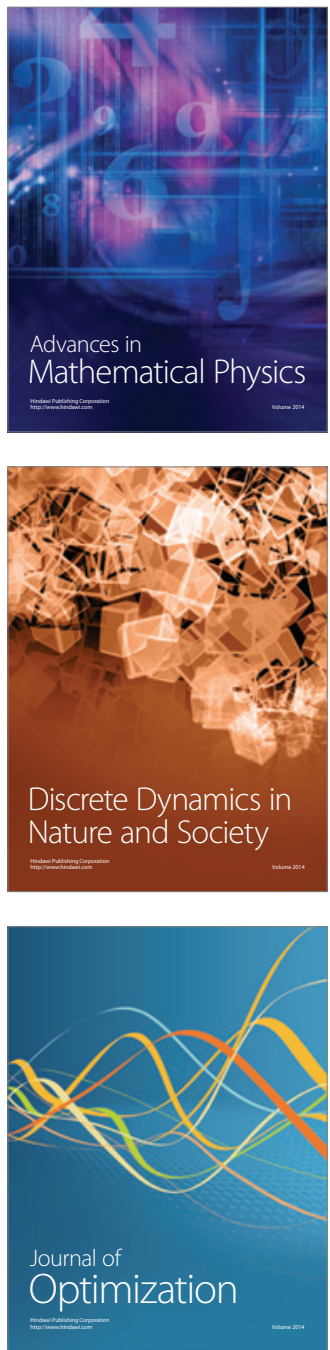